\title{
síndrome cardiometabólico?
}

Jorge NAVArro PÉrez

Médico de familia. Director Médico del Hospital Clínico Universitario de Valencia (Valencia), INCLIVA, Universitat de Valencia; CIBERESP, Red de Grupos de Estudio de la Diabetes en Atención Primaria de la Salud (redGDPS).

\begin{abstract}
Resumen
Las enfermedades cardiovasculares son la primera causa de mortalidad prematura en Europa. El síndrome metabólico o cardiometabólico es una asociación de factores de riesgo cardiovascular relacionados por un nexo fisiopatológico común, posiblemente la resistencia a la insulina.
\end{abstract}

Palabras clave: síndrome cardiometabólico, enfermedades cardiovasculares, obesidad.

\section{Epidemiología y diagnóstico}

Las enfermedades cardiovasculares siguen siendo la principal causa de muerte, seguidas por los tumores y las enfermedades respiratorias. Los factores de riesgo más importantes, por su prevalencia e impacto en la salud, son el tabaco, la presión arterial elevada, el índice de masa corporal elevado, el consumo de alcohol y la glucemia elevada. A causa del envejecimiento de la población, a pesar de que las tasas ajustadas de mortalidad por coronariopatía han ido disminuyendo, el número de muertes se ha incrementado, por lo que el impacto sanitario y social de estas enfermedades está aumentando. Las enfermedades cardiovasculares siguen siendo la primera causa de mortalidad prematura (< 70 años) en toda Europa. La hipertensión arterial, la dislipidemia y la diabetes son factores de riesgo mayores y causales de enfermedad cardiovascular y mortalidad vascular y total ${ }^{1}$.

En los últimos años se utiliza la expresión «pandemia» para hacer referencia tanto a la creciente prevalencia de obesidad en el mundo, como de síndrome metabólico o diabetes tipo 2.

En 1923 se definió el síndrome X como una asociación de hipertensión arterial, obesidad, hiperglucemia y gota. En 1927 Marañón habló de estados prediabéticos (Prädiabetische Zustände) refiriéndose a la hipertensión arterial y a la obesidad. En 1966 se encontró una asociación entre hipertensión arterial e hiperinsulinemia. En 1977 se emplea el término síndrome metabólico para referirse a la asociación de obesidad, diabetes e hígado graso, y a la asociación de obesidad, bocio, diabetes e hipertensión arterial. En 1988 se define como el síndrome X metabólico a la asociación de resistencia a la insulina, hiperinsulinemia, intolerancia a la glucosa, aumento de los niveles de triglicéridos, lipoproteínas de muy baja densidad (VLDL), disminución de lipoproteínas de alta densidad (HDL) e hipertensión arterial.

En 1998 la Organización Mundial de la Salud (OMS) propone una definición unificada del síndrome metabólico ${ }^{2}$. Buscando una diagnóstico más práctico, en 2001 el National Cholesterol Education Program $(\mathrm{NCEP})^{3}$ y en 2005 la International Diabetes Federation $(\text { IDF })^{4}$ propusieron versiones más prácticas, menos glucocéntricas.

El diagnóstico de síndrome metabólico se construye en torno a la existencia de resistencia a la insulina; es decir, en personas con diabetes tipo 2, intolerancia a la glucosa o alteración de la glucosa en ayunas (OMS 1998). Se consideraron parámetros principales o criterios mayores una glucemia en ayunas $>110 \mathrm{mg} / \mathrm{dl}$ y/o $>140$ mg/dl 2 h poscarga. Además, debían coexistir al menos dos de los siguientes criterios menores: presión arterial $\geq 140 / 90 \mathrm{mmHg}$, triglicéridos $\geq 150 \mathrm{mg} / \mathrm{dl}$, colesterol HDL $<35$ mg/dl en hombres o $<39$ mg/dl en mujeres, obesidad abdominal (perímetro de cintura $>102 \mathrm{~cm}$ en hombres y > 88 en mujeres, o índice de masa corporal $\left.(\mathrm{IMC})<30 \mathrm{~kg} / \mathrm{m}^{2}\right)$ o microalbuminuria $\geq 20 \mu \mathrm{g} / \mathrm{min}$. 
Tabla 1. Criterios diagnósticos de síndrome metabólico según OMS, NCEP e IDF.

\begin{tabular}{|c|c|c|c|}
\hline Componentes & $\begin{array}{l}\text { OMS } \\
1998^{a}\end{array}$ & $\begin{array}{l}\text { NCEP } \\
2001^{b}\end{array}$ & $\begin{array}{l}\text { IDF } \\
2005^{c}\end{array}$ \\
\hline Hiperglucemia (mg/dl) & $\begin{array}{l}G B A>110 \\
\text { o } 2 h-G P P>140\end{array}$ & $\mathrm{GBA}>100$ & $\mathrm{GBA}>100$ \\
\hline $\begin{array}{l}\text { Obesidad (perímetro de cintura, } \\
\text { índice de masa corporal) }\end{array}$ & $P C>102 / 88$ o IMC $>30$ & $P C>102 / 88$ & $P C>94 / 80$ \\
\hline Hipertensión arterial (mmHg) & $\geq 140 / 90$ & $>130 / 85$ & $>130 / 85$ \\
\hline Hipertrigliceridemia (mg/dl) & $\geq 150$ & $>150$ & $>150$ \\
\hline Colesterol HDL (mg/dl) & $<35 / 39$ & $>40 / 50$ & $>40 / 50$ \\
\hline Microalbuminuria ( $\mu \mathrm{g} / \mathrm{min})$ & $\geq 20$ & & \\
\hline
\end{tabular}

PC: perímetro de cintura; GBA: glucemia basal alterada; GPP: glucemia posprandial.

aHiperglucemia más otros dos criterios; ${ }^{\circ}$ Tres criterios de cinco; ' Obesidad más otros dos criterios.

El National Cholesterol Education Program (NCEP, 2001) estableció una nueva clasificación (ATP-III), más práctica, agrupando la coexistencia de factores de riesgo cardiovascular independientemente de la existencia de diabetes tipo 2. Comprendería la coexistencia de al menos tres de los siguientes factores: obesidad abdominal (perímetro de cintura $>102 \mathrm{~cm}$ en hombres y $>88$ en mujeres), triglicéridos ( $\geq 150 \mathrm{mg} / \mathrm{dl}$ o tratamiento farmacológico), c-HDL ( $<40 \mathrm{mg} / \mathrm{dl}$ en hombres $0<50 \mathrm{mg} / \mathrm{dl}$ en mujeres o tratamiento farmacológico), presión arterial alta (> 130/85 mmHg o tratamiento farmacológico) o hiperglucemia (> $100 \mathrm{mg} / \mathrm{dl}$ o tratamiento farmacológico). No obstante, a diferencia de lo que ocurría con la definición de la OMS, la definición ATP-III no incorporaba variables proinflamatorias ni protrombóticas como parte de una definición ampliada.

La IDF (2005) propone la obesidad abdominal como criterio principal o mayor, necesitándose para el diagnóstico de síndrome metabólico al menos otros dos criterios menores (PA $\geq 130 / 85$, triglicéridos $\geq 150 \mathrm{mg} / \mathrm{dl}$, $c-H D L<40$ o 50 mg/dl o glucemia en ayunas $\geq 100$ mg/ dl). Esta propuesta de la IDF contempla la obesidad central como un requisito necesario y en ella, por primera vez, se ofrecen valores umbral para definir la obesidad que son diferentes en los distintos grupos étnicos.

Dentro de cierta complejidad en el diagnóstico, se reconoce una clara asociación con la obesidad — sobre todo, abdominal- y con la diabetes tipo 2. La clasificación ATP-III detecta mayor número de personas con alto riesgo cardiovascular y la IDF detecta mayor número de personas con síndrome metabólico.

El síndrome metabólico se ha convertido en uno de los principales problemas de salud pública del siglo XXI.
Ha sido también identificado como un factor de riesgo independiente para las complicaciones cardiovasculares (cardiopatía isquémica, ictus, arteriopatía periférica, insuficiencia renal). Los mecanismos que asocian el síndrome metabólico con la aterosclerosis son múltiples: hipertrofia ventricular izquierda, proteína $C$ reactiva $(P C R)$, hipertrigliceridemia, hipoadiponectinemia. Además, síndrome metabólico y cardiopatía isquémica se relacionan a través de las alteraciones de la función renal. También se ha visto una correlación entre síndrome metabólico y la aparición de insuficiencia cardíaca, a través de la relación con la resistencia a la insulina o el cociente apolipoproteína B/apolipoproteína A (cociente apoB/A). Un estudio realizado en España muestra una asociación significativa entre la mortalidad por cardiopatía isquémica y la frecuencia de síndrome metabólico ${ }^{5}$.

También la obesidad se asocia con gran cantidad de marcadores séricos de inflamación; en concreto, la PCR predice la aparición de hipertensión arterial. También las alteraciones de la función renal (descenso del filtrado glomerular, microalbuminuria) se han relacionado con el desarrollo de hipertensión arterial, siendo el síndrome metabólico un factor de riesgo independiente de estas alteraciones renales.

La incidencia de síndrome metabólico evoluciona en paralelo a la de obesidad y a la de diabetes tipo 2. Una cuarta parte de la población adulta tendría síndrome metabólico ${ }^{6}$.

Según datos de National Health and Nutrition Examination Survey (NHNES), durante 1988-2010, el IMC promedio en los Estados Unidos (EE. UU.) aumentó un 0,37 \% por año, tanto en hombres como en mujeres, y 
el perímetro de cintura (PC) aumentó en un 0,37 y un $0,27 \%$ anual en hombres y mujeres, respectivamente. En 2017, alrededor de 30,2 millones mayores de 18 años, o el 12,2 \% de los adultos de EE. UU. tenían diabetes tipo 2. Una cuarta parte de estas personas $(23,8 \%)$ no eran conscientes de tener diabetes. La incidencia de diabetes tipo 2 aumenta con la edad, alcanzando un máximo del 25,2 \% entre las personas mayores de EE. UU. (65 años o más). La prevalencia de prediabetes o síndrome metabólico fue unas tres veces más. Aproximadamente, un tercio de los adultos de EE. UU. tiene síndrome metabólico ${ }^{7}$.

Se estima una prevalencia de síndrome metabólico del 12-37 \% en población asiática y del 12-26 \% en población europea. Recientes estudios confirman la especificidad del síndrome metabólico por sexo y población ${ }^{8}$. Un reciente metaanálisis ${ }^{9}$ muestra una clara asociación entre el síndrome metabólico y sus componentes con la mortalidad en el anciano, por cualquier causa (RR: 1,23; 95 \% Cl: 1,15-1,32) y cardiovascular (RR: 1,24, 1,11-1,39); de los componentes del síndrome metabólico, el mayor riesgo de mortalidad se asoció a niveles elevados de glucemia y a niveles bajos de CHDL.

En España se han realizado diversas aproximaciones al entorno del síndrome metabólico. Un estudio en pacientes hipertensos ancianos sin diabetes observó que la presencia de síndrome metabólico fue relacionada de forma independiente con una mayor prevalencia de lesión de órganos diana - hipertrofia ventricular izquierda (OR: 1,31; 95 \% Cl: 1,15-1,48), insuficiencia renal (OR: 1-45; 95 \% Cl: 1,29-1,63) y enfermedad cardiovascular establecida (OR: 1,22; $95 \%$ Cl: 1,08-1,37])— ${ }^{10}$. El estudio MEtabolic SYndrome in Active Subjects (MESYAS) muestra en población laboralmente activa una prevalencia bruta de SM del $10,2 \%$, significativamente superior en varones, que aumenta con la edad, la obesidad, la hipertensión y la diabetes ${ }^{11}$. Según el estudio Dyslipaemia, Atherosclerosis Risk and increased hsCRP and Inflammatory and Oxidative status in the Spanish population (DARIOS), la prevalencia de síndrome metabólico en adultos en España es del $31 \%$, algo mayor en varones que en mujeres (el 32 y el $29 \%$, respectivamente) y está asociado a mayor riesgo coronario ${ }^{12}$. El estudio prediabetes en Atención Primaria de Salud (PREDAPS) observa una clara asociación de obesidad abdominal principalmente con prediabetes, más que con hipertensión y dislipemia, más asociados con la obesidad general ${ }^{13}$.

\section{Fisiopatología}

El síndrome metabólico o cardiometabólico es una asociación de factores de riesgo cardiovascular relacionados por un nexo fisiopatológico común, posiblemente la resistencia a la insulina. Es altamente prevalente y guarda una estrecha relación con la obesidad.

El síndrome metabólico se asocia principalmente a obesidad, y esta al aumento del riesgo de desarrollar resistencia a la insulina y diabetes tipo 2. Tanto obesidad como diabetes tipo 2 se asocian a resistencia a la insulina, pero no todos los obesos resistentes a la insulina desarrollan hiperglucemia. Por un lado, las células beta pancreáticas tienen que ser incapaces de compensar el descenso de la sensibilidad a la insulina; asimismo, los ácidos grasos libres inducen resistencia a la insulina y descompensan la función de las células beta.

El tejido adiposo modula el metabolismo mediante la actuación de los ácidos grasos libres, glicerol y citosinas proinflamatorias. El aumento de niveles de ácidos grasos libres (observado en la obesidad y la diabetes tipo 2) se asocia a resistencia a la insulina. La distribución de grasa corporal observada en el síndrome metabólico es un determinante crítico de la sensibilidad a la insulina.

El diagnóstico busca, en esencia, seleccionar individuos con mayor riesgo cardiovascular, derivado de la disfunción metabólica conocida como resistencia a la insulina, y su repercusión en el metabolismo lipídico (disfunción del tejido adiposo). Existe alta correlación entre los índices de resistencia a la insulina (índices HOMA y QUICKI) y el nivel de triglicéridos, insulina basal y el cociente TG/HDL.

Al intentar comprender las bases moleculares del síndrome metabólico, los análisis factoriales muestran dos líneas de asociación diferentes. La que vincula obesidad e hipertensión, y la que vincula obesidad, dislipemia y alteraciones del control glucémico. Además, el riesgo cardiovascular depende más de la acumulación de factores que de la obesidad en sí misma.

La hipertensión en el síndrome metabólico debe ser entendida como una manifestación más de las alteraciones metabólicas debidas a la resistencia a la insulina. La aparición de hipertensión arterial se asocia a la presencia de determinadas dislipemias, la obesidad abdominal, el aumento sostenido del índice de masa corporal (IMC) o los marcadores séricos de inflamación. 
Las adipocinas (sustancias metabólicamente activas liberadas por el tejido adiposo) y, en concreto, los niveles bajos de adiponectina, se relacionan con la aparición de hipertensión arterial y síndromes coronarios agudos.

Se ha postulado una actividad endocrina del tejido adiposo, amortiguando los cambios lipídicos en los periodos posprandiales, modulando un equilibrio entre la captación de ácidos grasos libres procedentes de lipoproteínas ricas en triglicéridos durante el periodo posprandial y su liberación paulatina durante el ayuno. Por tanto, el ciclo homeostático propio del tejido adiposo se solapa con el del almacenamiento energético. Las adipocinas poseerían funciones de homeostasis. La leptina señaliza que el organismo tiene suficiente energía para vivir. La adiponectina retira los ácidos grasos libres hacia los depósitos de grasa ${ }^{14}$.

También se ha descrito en el tejido adiposo una autorregulación vascular. El incremento de flujo que sigue a la ingesta ocurre antes del pico plasmático de triglicéridos y paralelo al de la insulina.

El perfil dislipémico característico del síndrome metabólico respondería a un fallo en la función del tejido adiposo en la captación de grasa procedente de la dieta (estado permanente de liberación de ácidos grasos libres), que conduce a un exceso de flujo lipídico hacia otros tejidos durante el periodo posprandial junto a una disminución en el aclaramiento de las partículas ricas en triglicéridos. El hígado, estimulado por los ácidos grasos libres, los empaqueta en triglicéridos y los libera en lipoproteínas con ApoB en su superficie, que interaccionan con HDL y LDL. La leptina -que limita el depósito graso al disminuir la ingesta- está elevada en los sujetos obesos.

Alteraciones metabólicas como la dislipemia, la hiperinsulinemia, la resistencia a la insulina y la obesidad, juegan un papel clave en la inducción y progresión de la diabetes tipo 2. Asimismo, se ha establecido una implicación bidireccional entre los sistemas metabólico e inmunológico, jugando la inflamación un papel esencial en la promoción de alteraciones metabólicas. La infiltración celular inmune relacionada con la obesidad, la inflamación y el incremento de estrés oxidativo promueven alteraciones metabólicas en los tejidos sensibles a la insulina, llevando a la resistencia a la misma ${ }^{15}$. La diabetes tipo 2 está caracterizada por una enfermedad inflamatoria sistémica acompañada de resistencia a la insulina o respuesta metabólica reducida a la insulina. En personas obesas, el tejido adiposo expandido en diversas localizaciones, iniciando y perpetuando la inflamación, induce un estado inflamatorio crónico que promueve resistencia a la insulina.

En la asociación de la obesidad con la insulinorresistencia, el síndrome metabólico y el aumento del riesgo cardiovascular subyace un estado proinflamatorio crónico. Muchos de los marcadores inflamatorios son secretados por el tejido adiposo: las citoquinas (leptina, resistina, adiponeptina) y otras sustancias como el inhibidor del activador del plasminógeno (PAI-1), el

Figura 1. Fisiopatología del síndrome metabólico.

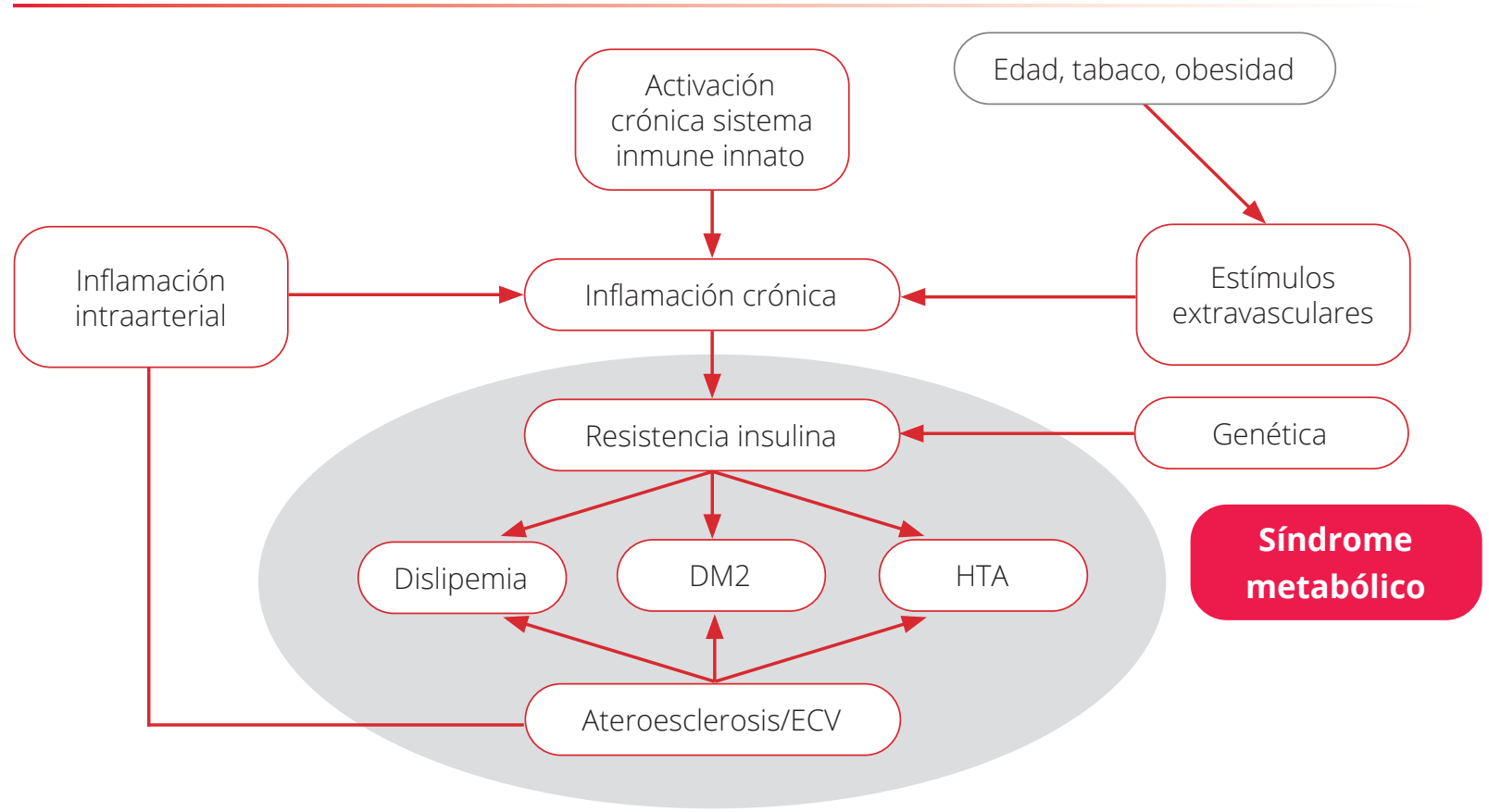


factor de necrosis tumoral alfa (TNF- $\alpha$ ) o la interleuquina tipo 6 (IL-6). Por ello, la aterosclerosis debe ser entendida como un proceso inflamatorio, secundario a la activación crónica del sistema inmune innato.

Alterados los mecanismos de regulación, los tejidos sobrecargados expresan una respuesta inflamatoria. El TNF- $\alpha$ y la IL-6 están aumentados en las personas obesas. La inflamación crónica contribuye al desequilibrio vascular y endotelial, ayudando al desarrollo de la enfermedad aterosclerótica. En las personas con obesidad la vasodilatación posprandial está disminuida, debido a la alteración de la vasodilatación dependiente de insulina, mediada por óxido nítrico.

Se ha observado una clara relación entre TNF- $\alpha$ y la IL-6 con hipertensión, dislipemia, obesidad abdominal, alteraciones de la coagulación y la acción de la insulina. Frente al resto, la adiponetina humana se correlaciona negativamente, tanto con la masa adiposa, como con la severidad de la resistencia a la insulina, presentando propiedades antiaterogénicas y antiinflamatorias. La adiponectina incrementa la sensibilidad a la insulina así como la funcionalidad y supervivencia de la célula beta pancreática, siendo protectora frente al síndrome metabólico.

Los macrófagos residentes en el tejido graso juegan un papel principal en el metabolismo energético. Los macrófagos proinflamatorios (M1) promueven esteatosis hepática y adipogénesis, mientras que los macrófagos antiinflamatorios (M2) hacen lo contrario.

El exceso de nutrientes produce inflamación sistémica en la diabetes que contribuye a la resistencia a la insulina, dislipidemia y aumento del riesgo cardiovascular. La inflamación es la respuesta de supervivencia de un organismo cuando se enfrenta a una infección, lesión o toxinas ${ }^{16}$. Si la agresión continúa, como en el exceso de nutrientes o metabolitos, la inflamación de bajo grado siempre permanece encendida con la activación de la respuesta inmune adaptativa, lo que da como resultado lo que ahora conocemos como inflamación sistémica. Dicha inflamación puede estar mediada por citocinas como TNF- $\alpha$, hormonas como la leptina, los propios lípidos, el estrés del retículo endoplásmico (RE) y la producción de reactive oxygen species (ROS) debido al exceso de glucosa, y es identificable por marcadores como CRP e IL-10, que son elevados en diabetes y obesidad. El exceso de nutrientes que conduce a una sobrecarga metabólica crónica está ahora bien establecido como una fuente de inflamación sistémica crónica en enfermedades relacionadas con la obesidad como DM2, aterosclerosis, enfermedad del hígado graso no alcohólico (NAFLD) e inflamación de las vías respiratorias.

¿Existe la obesidad metabólicamente sana? Los estudios revelan que la obesidad tiene un fenotipo heterogéneo y que no todas las personas obesas tienen el mismo riesgo de complicaciones. Los posibles mecanismos que potencialmente explicarían este estado son: variantes genéticas (mutaciones con pérdida de función), inflamación crónica reducida, cambios en la composición del tejido adiposo, variantes de la función de los adipocitos, protección frente al hígado graso no-alcohólico (NAFLD), variaciones en la microbiota gastrointestinal, estilo de vida menos sedentario (fat and fit) ${ }^{17}$.

Niveles elevados de la hormona incretina GLP-1 se asocian con componentes de síndrome metabólico en pacientes de alto riesgo con enfermedad cardiovascular. Los niveles en ayunas de GLP-1 en suero en la circulación periférica aumentaron significativamente en correlación con la acumulación de componentes de factores de riesgo de síndrome metabólico (riesgo relativo $=0,470, P<0,001)$. Hubo una interacción significativa entre el GLP-1 circulante y el polipéptido inhibidor gástrico (GIP), las concentraciones séricas de colesterol de lipoproteínas de alta densidad, triglicéridos y ácido úrico en suero, pero no la circunferencia de la cintura, la glucosa en ayunas, la $\mathrm{HbA}_{1 c}$ o la presencia de diabetes. Los niveles circulantes de GLP-1 en relación con la acumulación en factores de síndrome metabólico sugieren que los pacientes con síndrome metabólico con niveles elevados de GLP-1 son pacientes de alto riesgo de enfermedad cardiovascular, independientemente de la presencia de diabetes ${ }^{18}$.

De hecho, varios estudios en modelos animales han demostrado beneficios con los agonistas del GLP-1 y los inhibidores de la DPP-4 en la función endotelial, aterosclerosis y esteatosis hepática. Los análogos de GLP-1 (arGLP-1) reducen los marcadores de inflamación sistémica en personas con obesidad o diabetes tipo 2. Los arGLP-1 reducen la inflamación en células endoteliales y el estrés oxidativo inducido por el TNF- $\alpha^{19}$. También se han demostrado efectos antiinflamatorios favorables por la acción del arGLP-1 sobre los adipocitos. Estos efectos incluyen un aumento en la secreción de adiponectina de los adipocitos y un cambio en el fenotipo de macrófagos en el tejido adiposo de proinflamatorio a antiinflamatorio (cambio de M1 a M2). La infiltración de macrófagos en el tejido adiposo blanco también disminuye con el arGLP-1. 
Un estudio en ratones Apoe-/-Irs2 +/- muestra que los arGLP-1 reducen el tamaño y la inestabilidad de las placas de ateroma al reprogramar los macrófagos hacia un fenotipo M2, lo que conduce a una inflamación reducida, proporcionando evidencia experimental de un novedoso mecanismo de acción en la reducción del riesgo cardiovascular asociado con la resistencia a la insulina ${ }^{20}$.

Los componentes del síndrome metabólico (diabetes, hipertensión, obesidad) incrementan el riesgo

\section{Bibliografía}

1. Orozco-Beltrán D, Brotons Cuixart C, Alemán Sánchez JJ, Banegas Banegas JR, Cebrián-Cuenca AM, Gil Guillén VF, Martín Rioboó E, Navarro Pérez J. Recomendaciones preventivas cardiovasculares. Actualización PAPPS 2020. Aten Primaria. 2020;52(S2):5---31.

2. Alberti K, Zimmet P, for the WHO Consultation. Definition, diagnosis and classification of diabetes mellitus and its complications. Diabet Med 1998;15:539-553.

3. Executive summary of the third report of the National Cholesterol Education Program (NCEP). Expert panel on detection, evaluation and treatment of high blood colesterol in adults (Adult Treatment Panel III). JAMA 2001;285:2486-2497.

4. Alberti KGMM, Zimmet PZ, Shaw JE. The metabolic syndrome: a new world-wide definition from the International Diabetes Federation consensus. Lancet. 2005;366:1059-62.

5. León-Latre M, Andrés EM, Cordero A, Pascual I, Vispe C, Laclaustra M. Relación entre el síndrome metabólico y la mortalidad por cardiopatía isquémica en España. Rev Esp Cardiol 2009;62(12):1469-72.

6. Saklayen MG. The global epidemic of the metabolic síndrome. Current Hypertension Reports 2018;20:12.

7. National Center for Health Statistics, Division of Health Interview Statistics. Crude and age-adjusted percentage of civilian, noninstitutionalized adults with diagnosed diabetes, United States, 1980-2010. National Center for Chronic Disease Prevention and Health Promotion, Ed. Atlanta, GA, Centers for Disease Control and Prevention, Division of Diabetes Translation, 2012.

8. Sigit et al. The prevalence of metabolic síndrome and its association with body fat distribution in middle-aged individuals from Indonesia and the Netherlands: a cross-sectional analysis of two population-based studies.Diabetol Metab Syndr (2020) 12:2.

9. Sang-Yhun J, June-Young L, Do-Hoon K. Association of metabolic syndrome and its components with allcause and cardiovascular mortality in the elderly. A meta-analysis of prospective cohort studies. Medicine 2027; 96:45.

10. Navarro J, Redón J, Cea-Calvo L, Lozano JV, Fernández C, Bonet A., et al. Metabolic syndrome, organ damage and cardiovascular disease in treated hypertensive patients. The ERIC-HTA study. Blood Pressure 2007;16:20-27.

11. León M, Ordóñez B, Guallar E, Casasnovas JA. El síndrome metabólico en España: principales aportaciones del registro MESYAS y otros estudios. En Grima Serrano A (Coord). El síndrome metabólico. Soc Esp Cardiología, 2010. de hospitalización y mortalidad en pacientes con coronavirus (COVID-19). De nuevo, las alteraciones inmunitarias y el estado de sobreinflamación crónica explicarían esta mayor incidencia de morbimortalidad en pacientes COVID-1921, siendo claves la valoración de variables como el peso o los niveles de glucosa e insulina en la prevención de complicaciones severas asociadas a la COVID-1922.

12. Hernández-Bergés D, Cabrera de León A, Sanz H, Elosua R, Guembe MJ, Alzamora M, et al. Síndrome metabólico en España: prevalencia y riesgo coronario asociado a la definición armonizada y a la propuesta por la OMS. Estudio DARIOS. Rev Esp Cardiol 2012;65:241---8.

13. Sangrós FJ, Torrecilla J, Giráldez C, Carrillo L, Mancera J, et al. Asociación de obesidad general y abdominal con hipertensión, dislipemia y presencia de prediabetes en el estudio PREDAPS. Rev Esp Cardiol 2018;71(3):170-177.

14. Laclaustra M, Ordovás JM, Civeira F. Fisiopatología del síndrome metabólico: de la resistencia a la insulina y las adipocinas a la disfunción endotelial. En Grima Serrano A (Coord). El síndrome metabólico. Soc Esp Cardiología, 2010.

15. Daryabor G, Atashzar MR, Kabelitz D, Meri S, Kalantar K. The Effects of Type 2 Diabetes Mellitus on Organ Metabolism and the Immune System. Front. Immunol. 2020; 11:1582. doi: 10.3389/fimmu.2020.01582.

16. Hatwal A. Inflammation and incretins. Indian Journal of Endocrinology and Metabolism, vol 16, Supplement 2, S239-241.

17. Nilsson PM, Korduner JK, Magnusson M. Metabolically Healthy Obesity (MHO). New research directions for personalised medicine in cardiovascular prevention. Current Hypertension Reports 2020;22:18.

18. Yamaoka-Tojo et al. OElevated circulating levels of an incretin hormone, glucagon-like peptide-1, are associated with metabolic components in high-risk patients with cardiovascular disease Cardiovascular Diabetology 2010,9:17.

19. Shiraki A, Oyama J, Komoda H, Asaka M, Komatsu A, Sakuma $M$, et al. The glucagon-like peptide 1 analog liraglutide reduces TNF-a-induced oxidative stress and inflammation in endotelial cells. Atherosclerosis 2012, 221:375-382.

20. Vinué Á, Navarro J, Herrero-Cervera A, García-Cubas M, Andrés-Blasco I, Martínez-Hervás S, Real JT, Ascaso JF, González-Navarro H. The GLP-1 analogue lixisenatide decreases atherosclerosis in insulin-resistant mice by modulating macrophage phenotype. Diabetologia. 2017 Sep;60(9):1801-1812. doi: 10.1007/s00125-0174330-3. Epub 2017 Jun 12. PMID: 28608285.

21. Bansal R, Gubbi S, Muniyappa R. Metabolic Syndrome and COVID-19: Endocrine-Immune-Vascular Interactions Shapes Clinical Course. Endocrinology 2020 Oct 1;161(10):bqaa112. doi: 10.1210/endocr/bqaa112.

22. Stepfan N, Birkenfeld AL, Schulze MB, Ludwig DS. Obesity and impaired metabolic health in patients with COVID-19. Naure Reviews Endocrinology 2020. www. nature.com/nrendo. 